\title{
Solitary bone and brain metastasis in a patient with papillary thyroid carcinoma mimicking cavernous angioma
}

\author{
Eugene Han ${ }^{1}$, Ye Yeon Lee ${ }^{1}$, El Kim², Sun Young Kwon³, Sang Pyo Kim³, Hye Soon Kim², \\ and Mi Kyung Kim ${ }^{1}$
}

${ }^{1}$ Division of Endocrinology and Metabolism, Department of Internal Medicine, Departments of ${ }^{2}$ Neurosurgery and ${ }^{3}$ Pathology, Keimyung University School of Medicine, Daegu, Korea

\footnotetext{
Received: October 20, 2014

Revised : March 15, 2015

Accepted: April 12, 2015

\section{Correspondence to}

Mi Kyung Kim, M.D.

Division of Endocrinology and Metabolism, Department of Internal Medicine, Keimyung University School of Medicine, 56 Dalseong-ro, Jung-gu, Daegu 41931, Korea

Tel: +82-53-250-7486

Fax: +82-53-250-7982

E-mail:mdkmk@dsmc.or.kr
}

To the Editor,

There has been a recent increase in the global incidence rate of thyroid carcinoma. In Korea, this rate of increase is 23.3\% per year and, as a result, thyroid carcinoma has been the most common cancer in Korea since 2009 [1]. Papillary thyroid carcinoma (PTC) is the most common type of thyroid carcinoma and accounts for up to $80 \%$ of all newly diagnosed thyroid carcinoma cases. Although metastasis to local or regional lymph nodes can occur, distance metastasis is unusual. In particular, brain metastasis is very rare and is reported in only $1 \%$ of patients with thyroid carcinoma [2]. Here, we report a case of bone and brain metastasis in a patient with PTC in which the brain lesion mimicked cavernous angioma, a type of blood vessel malformation.

A 76-year-old woman with a history of diabetes mellitus presented with memory disturbances that had begun 3 months prior. The patient was cooperative but could not recall recent memories. A physical examination revealed a $5 \mathrm{~cm}$ mass without tenderness in the sternum but no palpable masses were detected in the thyroid gland. The vital signs of the patient were stable and apart from the presence of hyperglycemia (serum glucose level, $510 \mathrm{mg} / \mathrm{dL}$ ) the laboratory findings were all within normal limits. Brain magnetic resonance imaging (MRI) scans of the patient revealed a lobulated lesion in the right frontal lobe with a mixed signal core surrounded by a low intensity rim; the lesion was not enhanced in the contrast image. These particular MRI features are consistent with those of cavernous angioma with hemorrhage (Fig. $1 \mathrm{~A}$ and ${ }_{1 \mathrm{~B})}$.

A chest computed tomography (CT) scan was performed to assess the mass in the sternum and revealed a huge infiltrating tumor mass with necrosis extending into the manubrium (Fig. 1C). The patient underwent a craniotomy to remove the brain tumor and the manubrium mass was biopsied. A histological analysis of the brain mass under a low-power view revealed a cystic and solid tumor mass that was infiltrating into the brain parenchyma with hemorrhage (Fig. 2A and 2B) and a histological analysis under a high-power view revealed predominant branching papillary structures in the tumor that were composed of columnar cells with increased nuclear stratification (Fig. 2C). The sternum mass had identical histological features, i.e., the presence of complex papillary structures and equivalent cytological findings (Fig. 

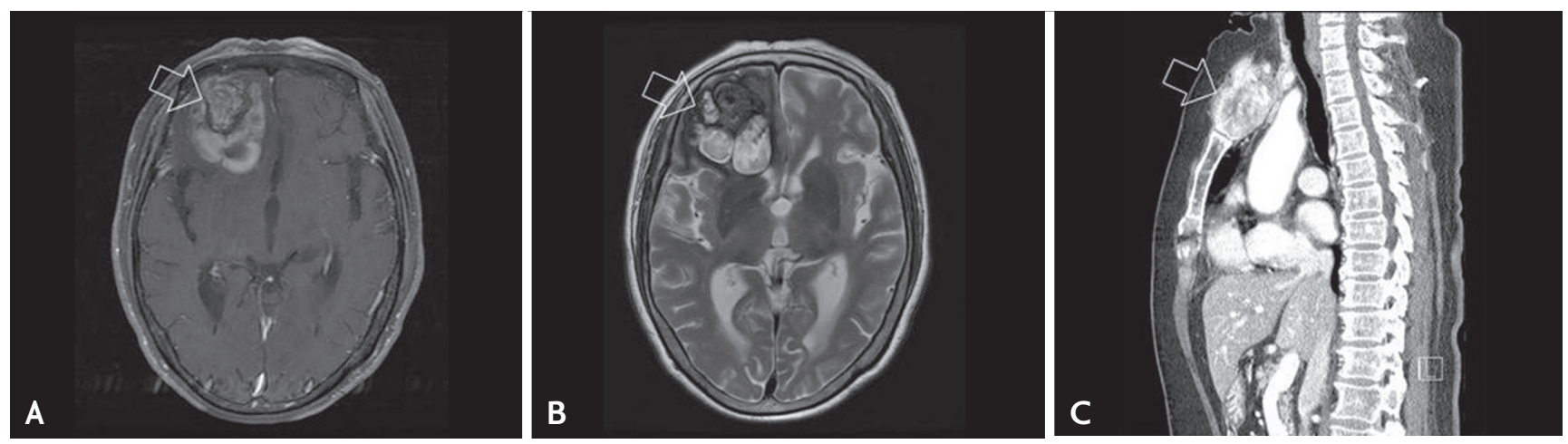

Figure 1. Radiological findings. (A) T1-weighted brain magnetic resonance image showing low mixed signal intensity (arrow). (B) T2-weighted image showing an isointense-to-hypointense lesion (arrow). (C) Chest computed tomography scan showing a 52 $\mathrm{mm}$ mass with necrosis on the manubrium of the sternum (arrow).

$2 \mathrm{D})$. To determine whether the sternum mass was a metastatic lesion from the lung, the mass was subjected to immunohistochemistry (IHC) analyses for cytokeratin 7, thyroid transcription factor-1 (TTF-1), and napsin A. The mass was positive for cytokeratin 7 and TTF- 1 but negative for napsin A (Fig. 2E-2G). Because metastatic thyroid cancer is characterized by a papillary structure and TTF-1 expression, the sternum mass was assessed for thyroglobulin expression and the IHC analysis revealed a strong expression of thyroglobulin (Fig. $2 \mathrm{H}$ ). The final pathological diagnosis was metastatic columnar cell-variant PTC, which is a very rare variant [3].

To evaluate the primary cancer, thyroid ultrasonography was performed. A single hypoechoic nodule $(5 \times 5 \times 10$ $\mathrm{mm}$ sized) with coarse calcification in the isthmus (Fig. $3 \mathrm{~A})$ and two indistinct subtle hyperechoic masses $(8 \times 8 \times$ $11 \mathrm{~mm}$ and $4 \times 5 \times 5 \mathrm{~mm}$ ) were detected in the right thyroid. All lymph nodes appeared normal and fine-needle aspiration of the isthmic thyroid nodule confirmed the diagnosis of PTC (Fig. 3B). A real-time polymerase chain reaction analysis revealed the presence of the BRAF mutation (V60oE) and a thyroid function test revealed a state of euthyroidism (thyroglobulin antibody [TgAb] concentrations > 1,000 ng/mL). To determine whether there were additional metastatic lesions, a positron emission tomography-CT scan was performed and revealed a low density and mildly hypermetabolic nodule in the right lobe of the thyroid gland (Fig. ${ }_{3} \mathrm{C}$ ) as well as a large infiltrating intensely hypermetabolic mass in the manubrium and upper sternum body (Fig. 3D).

Thyroidectomy and radiotherapy were recommended for the brain lesion but the patient refused all treatment.
Four months later, follow-up neck CT scans showed that the metastatic mass in the manubrium had undergone a marked increase in volume, the destruction of the sternum was evident, and there was new involvement of lymph nodes including an enlarged metastatic right level III lymph node that exhibited a probable invasion of the right internal jugular vein. A brain CT scan performed at the same time revealed newly developed meningeal metastasis and an intracranial hemorrhage in the right frontoparietal area. Five months after this follow-up visit, the patient expired due to pneumonia and sepsis.

In summary, the present 76-year-old female patient was initially diagnosed with cavernous angioma but her final diagnosis was thyroid cancer with brain and bone metastasis. In most cases, thyroid cancer has an excellent prognosis. In fact, the 5 -year relative survival rate for patients with thyroid cancer is almost $99.9 \%$ and the overall survival rate of patients with PTC is 98\% [1]. However, some histological variants of PTC are associated with an unusually poor prognosis including the conventional form, the follicular variant, the tall cell variant, the oncolytic variant, the columnar cell variant, the diffuse sclerosing variant, and the solid form. The present patient was diagnosed with the columnar cell variant, which is characterized by pseudostratified columnar cells and subnuclear vacuolization that resembles early secretory endometrium. Although this PTC variant is very rare and only accounts for $0.15 \%$ to $0.2 \%$ of all PTCs, it is associated with aggressive behaviors such as a propensity for extrathyroidal extension, distant metastasis, and the death of the tumor. Additionally, these tumor cells are usually positive for TTF-1 and show variable thyroglobulin im- 

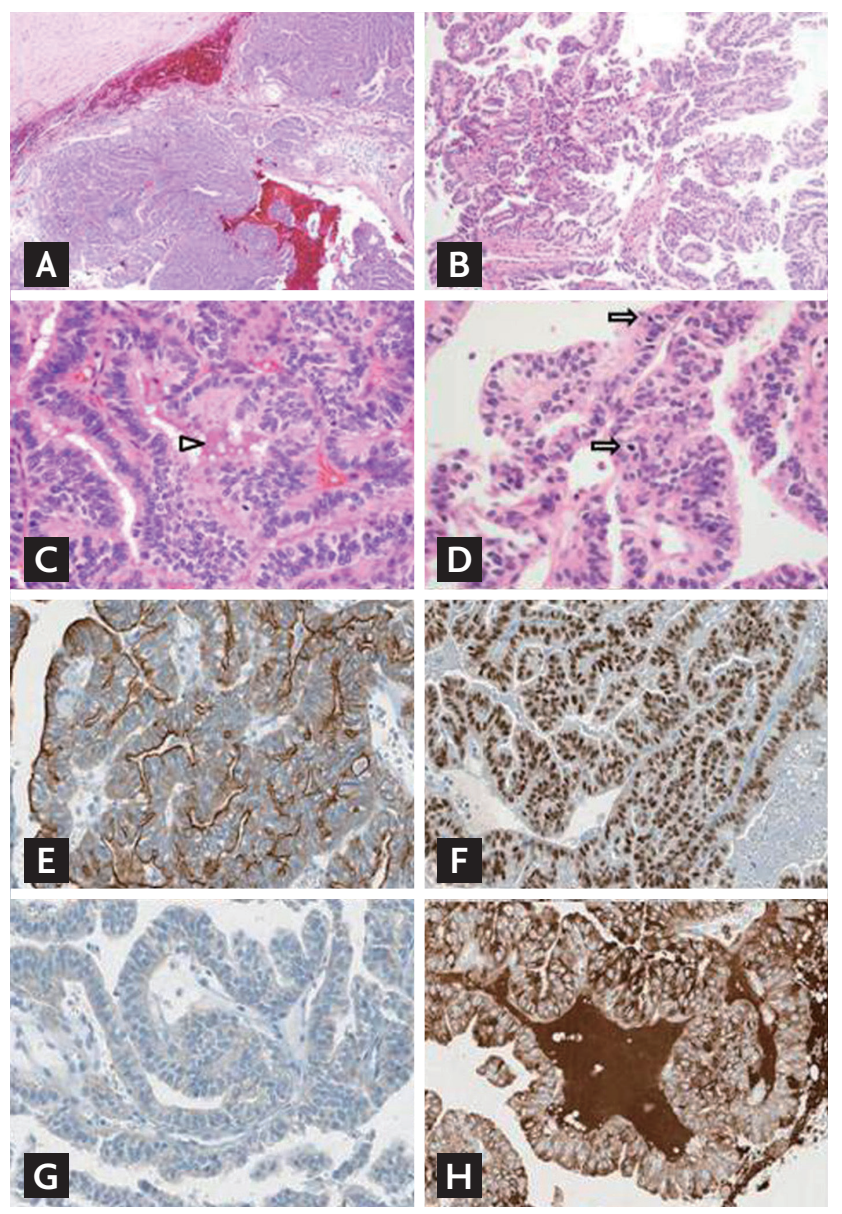

\section{G.}

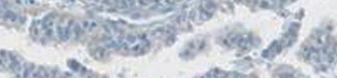

Figure 2. Microscopic findings of the brain and sternum tumor masses. (A) Low-power view of the resected right frontal mass showing a cystic and solid mass with hemorrhage (H\&E, $\times 10)$. (B) Low-power view of the brain mass revealing the branching and complex papillary structure of the tumor cells $(\mathrm{H} \& \mathrm{E}, \times 400)$. (C) High-power view of the brain mass showing the columnar atypical tumor cells with increased nuclear stratification. The eosinophilic colloid-like material within the lumen is also noted (arrowhead; H\&E, $\times 100$ ). (D) High-power view of the biopsied sternum mass showing the presence of histological features that were identical to the brain mass, i.e., complex papillary fronds and the same cytological findings. There are also increased mitotic figures (arrows; H\&E, ×400). Immunohistochemistry (IHC) for (E) cytokeratin $7,(\mathrm{~F})$ thyroid transcription factor-1 (TTF-1), (G) napsin A, and $(\mathrm{H})$ thyroglobulin. Unlike the classic IHC findings for metastatic adenocarcinomas from the lung (i.e., positivity for all three tissue-specific gene expression), the sternum mass expressed only cytokeratin 7 (E) and TTF-1 (F) but not napsin A (G, ×200). The IHC analysis also revealed that the sternum mass expressed thyroglobulin $(\mathrm{H}, \times 2 \mathrm{OO})$, which is consistent with a diagnosis of metastatic thyroid cancer.

munoreactivity but the typical nuclear features are not well-represented and may be mistaken for other kinds of
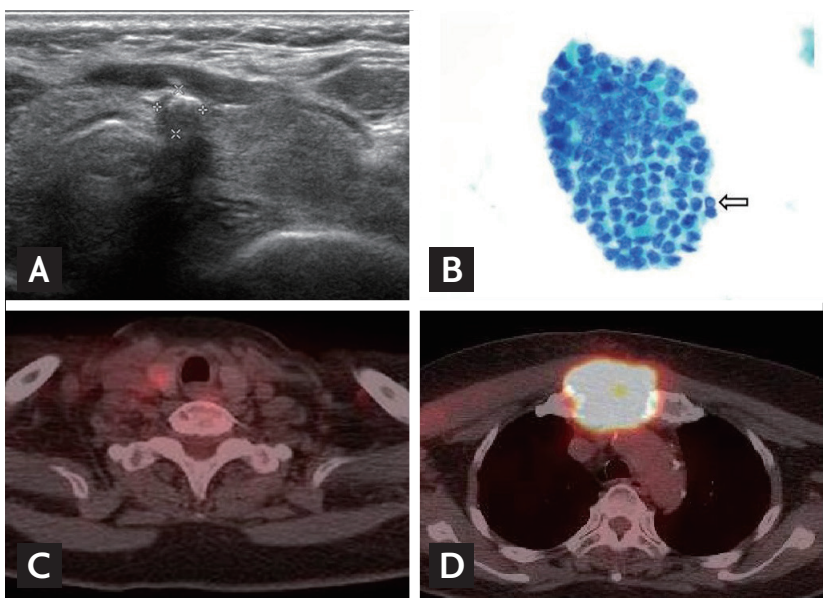

Figure 3. Findings from the thyroid ultrasonography, fine-needle aspiration, and positron emission tomography-computed tomography (PET-CT) scans. (A) Ultrasonography scan showing the presence of a $5 \times 5 \times 10 \mathrm{~mm}$ hypoechoic nodule with coarse calcification at the thyroid isthmus. (B) Fine-needle aspiration cytology of the thyroid gland showing the presence of angulated tumor cell nests with intranuclear grooves and prominent intranuclear pseudoinclusion (arrow; Pap smear, $\times 400$ ). (C) PET-CT scan revealing a low density and mildly hypermetabolic nodule in the right lobe of the thyroid gland. (D) PET-CT scan revealing a large infiltrating intensely hypermetabolic mass in the manubrium and upper sternal body.

adenocarcinomas [3].

Serum $\mathrm{TgAb}$ is a predictive marker of thyroid malignancy in thyroid nodules. The prevalence of $\mathrm{TgAb}$ in thyroid malignancy patients is $10 \%$ to $30 \%$, which is higher than among the normal population, and a high serum $\mathrm{TgAb}$ titer decreases after thyroid malignancy treatment while a sustained high serum $\mathrm{TgAb}$ titer could reflect early relapse [4]. The survival rate of PTC patients significantly decreases when metastasis occurs. The average overall survival duration of patients with PTC and brain metastasis is 4.7 months but if the PTC is well-differentiated with brain metastasis then the median survival duration is 12.4 months $[1,2]$. The common symptoms of patients with thyroid carcinoma and brain metastasis include headache, nausea, motor weakness, gait disturbances, sensory deficits, visual loss, and seizures but some patients with brain metastasis do not exhibit any symptoms and are only diagnosed with brain metastasis upon a postmortem examination. Among all PTC cases, the median interval between a diagnosis of PTC and the identification of brain metastasis is 2.9 years. Patients with brain metastasis from PTC tend to be older, have a large primary 
tumor size, and exhibit extrathyroidal invasion.

Due to the low incidence of brain metastatic PTC, a universal standard treatment protocol has yet to be established. However, the current treatment of choice is typically surgical resection because the removal of a brain metastasis significantly improves survival and is an independent predictor of better outcomes [2]. Radiosurgery should be considered for patients who are unable to undergo surgery and whole brain radiation therapy can also be used to treat multiple metastases. Although brain radiation is not associated with a survival benefit, several cases have been reported indicating a regression of brain metastasis after such therapy [5]. Similarly, there is no evidence of a treatment response to radioactive iodine or doxorubicin-based chemotherapy. Brain metastasis accounts for about $20 \%$ of cause-specific deaths in patients with PTC while other causes include pneumonia, sepsis, and local invasion [5].

The present case was an extremely rare example of brain metastasis from columnar cell-variant PTC in which the brain metastatic lesion mimicked a cavernous angioma. These findings suggest that, depending on the PTC variant, even small PTCs should be treated with caution because they can lead to brain metastasis.

Keywords: Brain; Neoplasm metastasis; Thyroid neoplasm

\section{Conflict of interest}

No potential conflict of interest relevant to this article was reported.

\section{REFERENCES}

1. Jung KW, Won YJ, Kong HJ, Oh CM, Lee DH, Lee JS. Cancer statistics in Korea: incidence, mortality, survival, and prevalence in 2011. Cancer Res Treat 2014;46:109123.

2. Chiu AC, Delpassand ES, Sherman SI. Prognosis and treatment of brain metastases in thyroid carcinoma. J Clin Endocrinol Metab 1997;82:3637-3642.

3. Sujoy V, Pinto A, Nose V. Columnar cell variant of papillary thyroid carcinoma: a study of 10 cases with emphasis on CDX2 expression. Thyroid 2013;23:714-719.

4. Kim ES, Lim DJ, Baek KH, et al. Thyroglobulin antibody is associated with increased cancer risk in thyroid nodules. Thyroid 2010;20:885-891.

5. McWilliams RR, Giannini C, Hay ID, Atkinson JL, Stafford SL, Buckner JC. Management of brain metastases from thyroid carcinoma: a study of 16 pathologically confirmed cases over 25 years. Cancer 2003;98:356-362. 